\title{
Is Blockchain Part of the Future of Art?
}

Stylianos Kampakis

University College London, UK

Correspondence: stylianos.kampakis@gmail.com

Received: 30 July 2019 Accepted: 13 August 2019 Published: 17 August 2019

Art is an important part of our culture, and economy. The global art market reached $\$ 67$ billion in $2018^{i}$. While some individuals might purchase art solely for their own enjoyment, for others it can be a status symbol or an investment.

However, the world of art is not without its problems. Two of the most important challenges are are fraud, and ownership of digital assets. However, blockchain is promising to solve both of these issues soon.

Fraud in artwork usually shows up in the form of forging. While it is not easy to estimate the total amount of money exchanged in forged art, it is clear that individuals and museums might be losing millions of dollars every year because of forging. In 2018, it was reported that a museum in France ${ }^{\mathrm{ii}}$ dedicated to the art of Étienne Terrus, discovered that most of the artworks were not real. There are also plenty other famous cases of forgery last year, such as that of an exhibition about Amedeo Modigliani in Genoa, where 21 of the 30 artworks were confirmed as fakes ${ }^{\text {iii }}$. While these paintings might have been worth millions of dollars (if they were authentic), the fakes were practically worthless.

These are only two of the forgery cases that took place in 2018. It is easy to find more examples, but what is shocking, is that a large part of the forgeries is never uncovered. It is possible that a forged artwork exchanges hands many times, until the final owner realizes that the actual value is zero.

Given blockchain's ability to help in the provenance of goods, it is a natural ally in the battle against art forgery. The problem of authenticity in art, is not different to the problem of provenance in supply chains. A work can be identified through a single identifier which can be, for example, an image hash, such as perceptual hashing. The ownership of the work can be stored on the blockchain. A smart contract or a Ricardian contract can be used in order to transfer ownership of the artwork.

There are different companies working on that problem right now, like Vastari and Thomas Crown Art. While no standard solutions have emerged, we are likely to see one in the next few years.

Another important problem that blockchain is aiming to solve, is the ownership of digital assets. While for physical assets the only problem is forgery, digital assets can be copied an unlimited number of times. Therefore, until blockchain came about, it was impossible to create digital collectibles.

The first instance of a blockchain-based collectible was the Rare Pepe Wallet, in 2016, based on an internet memeiv. However, the most monumental moment for crypto collectibles was the creation of Cryptokitties in 2017. Cryptokitties is by far the most successful game of crypto collectibles. In this game, the users own cats that have certain attributes, like colour or weird features like wings. The cats can mate with each other creating new cats with unique combinations of attributes. There are in total 4 billion cats that can be bred.

The game combines elements of collectible card games, with the breeding mechanism that could only exist inside a computer. The game reached a total number of 1 million transactions in October 2018.

At the time of writing, there are multiple exchanges for cryptocollectibles and blockchain-based artworks: opensea.io, digitalobjects.art, rareart.io, pixura.io, Known Origin, Maecenas and Makers Place are some of them. Artists can easily secure ownership their artworks on blockchain through Mintable or Pixura. On some of those exchanges, you can find collectibles 
and artwork that have reached higher price tags. Some artworks on Digital Objects can go up to $\$ 1000$. On Open Sea, there are collectibles that are sold for 10 ethers or more, which, at the time of writing, amounts to more than $\$ 3000$. Finally, a digital card of Elon Musk was recently sold for over $\$ 50,000$.

Ethereum has fully supported crypto-collectibles, through the ERC-721 standard. Much like the ERC-20 standard describes how to setup smart contracts for fungible tokens, the ERC-721 standard, describes how to setup a smart-contract for nonfungible tokens. That is, all tokens that are using this standard are unique. The aforementioned exchanges are all based on this standard.

So, to answer the question that was set out in the beginning of the article: Yes, blockchain is definitely going to play a key role in the future of art, and we saw in this short article two ways in which it is going to disrupt the world of art. It is clear that there are still some barriers to the widespread adoption of blockchain.

Cryptocurrency prices can still fluctuate rapidly, and the speculative bubble that burst in December 2018 might have

i https://www.artsy.net/article/artsy-editorial-global-art-market-reached-674billion-2018-6

ii https://www.theguardian.com/global/2019/jun/15/french-art-museum-full-offakes-etienne-terrus hurt the credibility and popularity of cryptocurrencies. Also, buying Ethereum and exchanging is something that is not easy for everyone. While in practice, tools like the Metamask Chrome extension or the Brave browser make it easy to use Ethereum, audiences that are less familiar with technology might find this challenging. Given that a large part of high networth art buyers might be of older age, this can become a significant barrier.

However, given the usefulness of blockchain and its rising popularity, we expect that in the next few years accessibility will increase, and use cases will multiply. Therefore, blockchain for art is here to stay.

Disclaimer: The author of this article is personally involved in this space, by using generative adversarial networks to create works of art and sell them on blockchain.

iii https://www.telegraph.co.uk/news/2018/01/10/modigliani-paintings-thoughtworth-tens-millions-denounced-fakes/

iv https:// rarepepewallet.com/ 